MMCA-97 Conference, pp. 92-104

R. Čiegis (Ed)

(C) 1997 Vilniaus Gedimino technikos universitetas

\title{
SOLUTION OF ABEL-TYPE HYPERGEOMETRIC INTEGRAL EQUATION
}

\author{
A.A.KILBAS, R.K.RAINA, M.SAIGO and H.M.SRIVASTAVA \\ Department of Mathematics and Mechanics, \\ Belarusian State University, \\ 220050 Minsk, Belarus \\ College of Technology and Agricultural Engineering, \\ Udaipur 313001, Rajasthan, India \\ Department of Applied Mathematics, \\ Fukuoka University, \\ Fukuoka 814-80, Japan \\ Department of Mathematics and Statistics, \\ University of Victoria, \\ Victoria, British Columbia, V8W 3P4, Canada
}

\section{ABSTRACT}

The paper is devoted to the study of the one-dimensional integral equation involving the Gauss hypergeometric function in the kernel. The necessary and sufficient conditions for the solvability of such an equation in the space of summable functions are proved and two forms for its solution are given.

\section{INTRODUCTION}

The present paper is devoted to the study of the integral equation

$$
\frac{(x-h)^{\alpha}}{\Gamma(\gamma)} \int_{a}^{x}(x-t)^{\gamma-1} F\left(\alpha, \beta ; \gamma ; \frac{x-t}{x-h}\right) \varphi(t) d t=f(x)(a<b)
$$

with $h \leq a, \alpha, \beta \in R$ and $\gamma \in R(0<\gamma<1)$ on a finite interval $(a, b)$ of the real axis $R=(-\infty, \infty)$. The kernel of this equation contains the Gauss 
hypergeometric function $F(a, b ; c ; z)[3]$ defined for $|z|<1$ by

$$
F(a, b ; c ; z) \equiv_{2} F_{1}(a, b ; c ; z)=\sum_{n=0}^{\infty} \frac{(a)_{n}(b)_{n}}{(c)_{n}} \frac{z^{n}}{n !}
$$

where $(z)_{n}$ is the Pochhammer symbol:

$$
(z)_{0}=1 ;(z)_{n}=z(z+1) \cdots(z+n-1)(n=1,2, \cdots) .
$$

The equation (3.1) genalizes the classical Abel integral equation [22, Section $2]$ being obtained from (1.1) in the case $\alpha=0$, because $F(0, \beta ; \gamma ; z)=1$. Therefore, such an equation is called the Abel-type hypergeometric integral equation.

One-dimensional Abel-type integral equations involving the Gauss hypergeometric function in the kernel have been studied by many authors [1], [4], [7]-[12], [17], [22, Section 35.1], [23], [24] - see also [25]. Such equations have arisen in the boundary value problems for partial differential equations with boundary conditions involving generalized integro-differentiation operators [5], [13]-[16], [18]-[20], [21], [24], [26], [27]. One such multidimensional integral equation of non-convolution type was investigated in [2, Section 4.6.2]. In the papers above, the integral operators of the equations considered were represented as compositions of simpler fractional integration operators with power weights. On the basis of these representations and the known properties of fractional calculus operators, the sufficient conditions for the solvability of the integral equations were given and their inversion formulas were obtained in some function spaces.

The investigation of necessary and sufficient conditions for the solvability of the above equations is more difficult. This problem is closely connected with characterization of the range of the corresponding integral operators. The classical Tamarkin's statement [22, Section 2.2] on the solvability of the Abel integral equation in the space $L_{1}(a, b)$ of summable functions is known. A similar result for the multidimensional Abel-type integral equation over pyramidal domain was proved in [6].

Our paper is devoted to obtain the aformentioted results for the integral equation (1.1) which was investigated in [17] in the particular case when $h=a$, with $\alpha, \beta$ and $\gamma$ being replaced by $\alpha+\beta,-\eta$ and $\alpha$, respectively. Solution of the equation (1.1) is given in Section 2. A preliminary lemma is proved in Section 3. Section 4 deals with the solvability of this equation in $L_{1}(a, b)$. Section 5 is devoted to obtain sufficient conditions for the solvability of the equation (1.1) and another form for its solution.

\section{SOLUTION OF THE INTEGRAL EQUATION}

We suppose that the integral equation (1.1) is solvable and find its solution $\varphi(x)$ in the following way. Replacing $x$ by $t$ and $t$ by $\tau$ and multiplying both 
sides of the resulting equation by $(x-t)^{-\gamma} F\left(-\alpha, 1+\beta-\gamma ; 1-\gamma ; \frac{x-t}{x-h}\right)$ and then integrating over $(a, x)$, we obtain

$$
\begin{aligned}
& \int_{a}^{x}(x-t)^{-\gamma} F\left(-\alpha, 1+\beta-\gamma ; 1-\gamma ; \frac{x-t}{x-h}\right)(t-h)^{-\alpha} d t \\
& \cdot \int_{a}^{t}(t-\tau)^{\gamma-1} F\left(\alpha, \beta ; \gamma ; \frac{t-\tau}{t-h}\right) \varphi(\tau) d \tau \\
&=\Gamma(\gamma) \int_{a}^{x}(x-t)^{-\gamma} F\left(-\alpha, 1+\beta-\gamma ; 1-\gamma ; \frac{x-t}{x-h}\right) f(t) d t .
\end{aligned}
$$

Interchanging the order of integration at the left-hand side of (2.1), and making the change of variables $t=\tau+(1-s)(x-\tau)$, we rewrite the left-hand side of (2.1) in the form:

$$
\begin{gathered}
(x-h)^{-\alpha} \int_{a}^{x} \varphi(\tau) d \tau \int_{0}^{1} s^{-\gamma}(1-s)^{\gamma-1}\left(1-s \frac{x-\tau}{x-h}\right)^{-\alpha} \\
\cdot F\left(\alpha, \beta ; \gamma ; \frac{(1-s)(x-\tau) /(x-h)}{1-s[(x-\tau) /(x-h)]}\right) F\left(-\alpha, 1+\beta-\gamma ; 1-\gamma ; s \frac{x-\tau}{x-h}\right) d s .
\end{gathered}
$$

By using $[3,2.4(3)]$ the latter is seen to be equal to

$$
\begin{gathered}
B(\gamma, 1-\gamma)(x-h)^{-\alpha} \int_{a}^{x} F\left(0,1+\beta-\gamma ; 1 ; \frac{x-\tau}{x-h}\right) \varphi(\tau) d \tau \\
=\Gamma(\gamma) \Gamma(1-\gamma)(x-h)^{-\alpha} \int_{a}^{x} \varphi(\tau) d \tau
\end{gathered}
$$

and therefore (2.1) is rewriten as

$$
\int_{a}^{x} \varphi(\tau) d \tau=\frac{(x-h)^{\alpha}}{\Gamma(1-\gamma)} \int_{a}^{x}(x-t)^{-\gamma} F\left(-\alpha, 1+\beta-\gamma ; 1-\gamma ; \frac{x-t}{x-h}\right) f(t) d t .
$$

It follows from (2.2) that the solution $\varphi(x)$ of the equation (1.1) has the form:

$$
=\frac{1}{\Gamma(1-\gamma)} \frac{d}{d x}\left[(x-h)^{\alpha} \int_{a}^{x}(x-t)^{-\gamma} F\left(-\alpha, 1+\beta-\gamma ; 1-\gamma ; \frac{x-t}{x-h}\right) f(t) d t\right] .
$$




\section{A PRELIMINARY LEMMA}

To obtain the solvability conditions of the equation (1.1) in the space $L_{1}(a, b)$, we put

$$
f_{h}^{\gamma, \alpha, \beta}(x)=\frac{(x-h)^{\alpha}}{\Gamma(1-\gamma)} \int_{a}^{x}(x-t)^{-\gamma} F\left(-\alpha, 1+\beta-\gamma ; 1-\gamma ; \frac{x-t}{x-h}\right) f(t) d t .
$$

The following preliminary assertion holds.

Lemma 1. Let $f(t) \in L_{1}(a, b), h \leq a, \alpha, \beta \in R, 0<\gamma<1$. If

$$
f(t)=O\left((t-a)^{\mu}\right), \mu>-1(t \rightarrow a)
$$

when $h<a$ or

$$
f(t)=O\left((t-a)^{\mu}\right), \mu>\max [\beta-\alpha, 0]-1(t \rightarrow a),
$$

when $h=a$, and

$$
f(t)=O\left((b-t)^{\nu}\right), \nu>\gamma-2(t \rightarrow b),
$$

then $f_{h}^{\gamma, \alpha, \beta}(x) \in L_{1}(a, b)$.

$\mathrm{P} \mathrm{r}$ o o f. Using (3.1) and interchanging the order of integration, we have

$$
\begin{gathered}
\int_{a}^{b} f_{h}^{\gamma, \alpha, \beta}(x) d x= \\
=\frac{1}{\Gamma(1-\gamma)} \int_{a}^{b} f(t) d t \int_{t}^{b}(x-h)^{\alpha}(x-t)^{-\gamma} F\left(-\alpha, 1+\beta-\gamma ; 1-\gamma ; \frac{x-t}{x-h}\right) d x .
\end{gathered}
$$

To evaluate the inner integral, we apply the series expansion (1.2), make the change of variables $\tau=(x-t) /(b-t)$, and by using the integral representation for the Gauss hypergeometric function [3, 2.1(10)] and (1.3), we obtain

$$
\begin{aligned}
I= & \int_{t}^{b}(x-h)^{\alpha}(x-t)^{-\gamma} F\left(-\alpha, 1+\beta-\gamma ; 1-\gamma ; \frac{x-t}{x-h}\right) d x \\
= & \sum_{k=0}^{\infty} \frac{(-\alpha)_{k}(1+\beta-\gamma)_{k}}{(1-\gamma)_{k} k !} \int_{t}^{b}(x-h)^{\alpha-k}(x-t)^{-\gamma+k} d x \\
& =\sum_{k=0}^{\infty} \frac{(-\alpha)_{k}(1+\beta-\gamma)_{k}}{(1-\gamma)_{k} k !}(t-h)^{\alpha-k}(b-t)^{1-\gamma+k}
\end{aligned}
$$




$$
\begin{gathered}
\cdot \int_{0}^{1} \tau^{-\gamma+k}\left[1-\left(-\frac{b-t}{t-h}\right) \tau\right]^{\alpha-k} d \tau \\
=\frac{(t-h)^{\alpha}(b-t)^{1-\gamma}}{1-\gamma} \sum_{k=0}^{\infty} \frac{(-\alpha)_{k}(1+\beta-\gamma)_{k}}{(2-\gamma)_{k} k !}\left(\frac{b-t}{t-h}\right)^{k} \\
\cdot F\left(k-\alpha, 1-\gamma+k ; 2-\gamma+k ;-\frac{b-t}{t-h}\right)
\end{gathered}
$$

where we have also used the relations

$$
(-\alpha)_{k}(-\alpha+k)_{m-k}=(-\alpha)_{m},(2-\gamma)_{k}(2-\gamma+k)_{m-k}=(2-\gamma)_{m}
$$

Applying again (1.2), and changing the order of summation $k+j=m$, in accordance with (1.3), we have

$$
\begin{gathered}
I=\frac{(t-h)^{\alpha}(b-t)^{1-\gamma}}{1-\gamma} \sum_{k=0}^{\infty} \frac{(-\alpha)_{k}(1+\beta-\gamma)_{k}}{(2-\gamma)_{k} k !}\left(\frac{b-t}{t-h}\right)^{k} \\
\cdot \sum_{j=0}^{\infty} \frac{(k-\alpha)_{j}(1+k-\gamma)_{j}}{(2+k-\gamma)_{j} j !}\left(-\frac{b-t}{t-h}\right)^{j}=\frac{(t-h)^{\alpha}(b-t)^{1-\gamma}}{1-\gamma} \sum_{m=0}^{\infty}\left(\frac{b-t}{t-h}\right)^{m} \\
\cdot \sum_{k=0}^{m}(-1)^{m-k} \frac{(-\alpha)_{k}(-\alpha+k)_{m-k}(1+\beta-\gamma)_{k}(1+k-\gamma)_{m-k}}{(2-\gamma)_{k}(2+k-\gamma)_{m-k} k !(m-k) !} \\
=\frac{(t-h)^{\alpha}(b-t)^{1-\gamma}}{1-\gamma} \sum_{m=0}^{\infty} \frac{(-\alpha)_{m}}{(2-\gamma)_{m}}\left(\frac{b-t}{t-h}\right)^{m} \\
\cdot \sum_{k=0}^{m}(-1)^{m-k} \frac{(1+\beta-\gamma)_{k}(1+k-\gamma)_{m-k}}{k !(m-k)_{!}}
\end{gathered}
$$

Using the relations

$$
\begin{gathered}
(1-\gamma+k)_{m-k}=\frac{(1-\gamma)_{m}}{(1-\gamma)_{k}},(m-k) !=(-1)^{m-k} \frac{(-m)_{m}}{(-m)_{k}} \\
F(\alpha, \beta ; \gamma ; 1)=\frac{\Gamma(\gamma) \Gamma(\gamma-\alpha-\beta)}{\Gamma(\gamma-\alpha) \Gamma(\gamma-\beta)}(\operatorname{Re}(\gamma-\alpha-\beta)>0)
\end{gathered}
$$


and (1.2) and (1.3), we evaluate the inner sum in (3.6):

$$
\begin{aligned}
& \sum_{k=0}^{m}(-1)^{m-k} \frac{(1+\beta-\gamma)_{k}(1+k-\gamma)_{m-k}}{k !(m-k) !}= \\
& =\frac{(1-\gamma)_{m}}{(-m)_{m}} F(1+\beta-\gamma,-m ; 1-\gamma ; 1)=\frac{(1-\gamma)_{m}}{(-m)_{m}} \frac{\Gamma(1-\gamma) \Gamma(-\beta+m)}{\Gamma(1-\gamma+m) \Gamma(-\beta)} \\
& =\frac{(-\beta)_{m}}{(-m)_{m}}=\frac{(-1)^{m}(-\beta)_{m}}{m !} .
\end{aligned}
$$

Substituting this relation in (3.6), we have

$$
I=\frac{(t-h)^{\alpha}(b-t)^{1-\gamma}}{1-\gamma} F\left(-\alpha,-\beta ; 2-\gamma ;-\frac{b-t}{t-h}\right) .
$$

Then, from (3.5) and (3.6), we find that

$$
\begin{gathered}
\int_{a}^{b} f_{h}^{\gamma, \alpha, \beta}(x) d x=\int_{a}^{b} F_{h}^{\gamma, \alpha, \beta}(t) d t \\
F_{h}^{\gamma, \alpha, \beta}(t)=\frac{1}{\Gamma(2-\gamma)}(t-h)^{\alpha}(b-t)^{1-\gamma} F\left(-\alpha,-\beta ; 2-\gamma ;-\frac{b-t}{t-h}\right) f(t) .
\end{gathered}
$$

When $h<a$, by $(3.2), F_{h}^{\gamma, \alpha, \beta}(t)=O\left((t-a)^{\alpha+\mu}\right)$ as $x \rightarrow a$. If $h=a$, according to (3.3) and asymptotics of the Gauss hypergeometric function (1.2) at infinity [3, 2.3(9)], we obtain the asymptotic behaviour of $F_{h}^{\gamma, \alpha, \beta}(t)$ near $t=a$ :

$$
F_{h}^{\gamma, \alpha, \beta}(t)=O\left((t-a)^{\mu}\right)+O\left((t-a)^{\mu+\alpha-\beta}\right)(t \rightarrow a)
$$

in the case of noninteger $\alpha-\beta$ with addition of $\log (t-h)$ for the case of integer $\alpha-\beta$. Equation (3.4) gives the asymptotics near $t=b$ :

$$
F_{h}^{\gamma, \alpha, \beta}(t)=O\left((b-t)^{\nu+1-\gamma}\right)(t \rightarrow b) .
$$

So $f_{h}^{\gamma, \alpha, \beta}(x)$ is integrable on $(a, b)$, and by (3.8) we have

$$
\begin{gathered}
\int_{a}^{b}\left|f_{h}^{\gamma, \alpha, \beta}(x)\right| d x \leq \frac{1}{\Gamma(2-\gamma)} \\
\int_{a}^{b}(t-h)^{\alpha}(b-t)^{1-\gamma}\left|F\left(-\alpha,-\beta ; 2-\gamma ;-\frac{b-t}{t-h}\right)\right||f(t)| d t<\infty .
\end{gathered}
$$

Hence $f_{h}^{\gamma, \alpha, \beta}(x) \in L_{1}(a, b)$. This completes the proof of the lemma. 


\section{SOLVABILITY OF THE INTEGRAL EQUATION}

We denote by $A C[a, b]$ the space of absolutely continuous functions on $[a, b]$. It is known (see, for example, [22, Section 1.1]) that this space coincides with the space of primitives of Lebesgue summable functions on $[a, b]$, namely

$$
A C[a, b]=\left\{f: f(x)=c+\int_{\alpha}^{x} g(t) d t, \int_{a}^{b}|g(t)| d t<\infty .\right.
$$

THEOREM 1. Let $\alpha, \beta$ and $0<\gamma<1$ be real numbers and let $f_{h}^{\gamma, \alpha, \beta}(x)$ $(h \leq a)$ be given by (3.1). The Abel-type hypergeometric integral equation (1.1) is solvable in $L_{1}(a, b)$ if and only if

$$
f_{h}^{\gamma, \alpha, \beta}(x) \in A C([a, b]), f_{h}^{\gamma, \alpha, \beta}(a)=0 .
$$

Under these conditions, the equation (1.1) has a unique solution given by (2.3).

$\mathrm{P}$ r o o f. To prove the neccesity part, let (1.1) be solvable in $L_{1}(a, b)$. Then all steps described in Section 2, in which the change of order of integration in (2.1) is justified by Fubini's theorem, are true. Thus (2.2) is valid. Hence (4.2) follows from (4.1).

To prove the sufficiency part, let the conditions in (4.2) hold. Then

$$
\left(f_{h}^{\gamma, \alpha, \beta}(x)\right)^{\prime}=\frac{d}{d x} f_{h}^{\gamma, \alpha, \beta}(x) \in L_{1}(a, b),
$$

in view of (3.8) and (3.9). We show that it is a solution of (1.1). Substituting $\varphi(x)$ from (2.3) into the left-hand side of (1.1) and denoting the resulting expression by $g(x)$, we have

$$
\frac{(x-h)^{-\alpha}}{\Gamma(\gamma)} \int_{a}^{x}(x-t)^{\gamma-1} F\left(\alpha, \beta ; \gamma ; \frac{x-t}{x-h}\right)\left(f_{h}^{\gamma, \alpha, \beta}(t)\right)^{\prime} d t=g(x) .
$$

This is an integral equation of the form (1.1) involving the prescribed function $\left(f_{h}^{\gamma, \alpha, \beta}(x)\right)^{\prime}$. It is certainly solvable, and so by $(2.3)$, we have

$$
\left(f_{h}^{\gamma, \alpha, \beta}(x)\right)^{\prime}=\left(g_{h}^{\gamma, \alpha, \beta}(x)\right)^{\prime}
$$

where $g_{h}^{\gamma, \alpha, \beta}(x)$ is expressed similarly to (3.1):

$$
g_{h}^{\gamma, \alpha, \beta}(x)=\frac{(x-h)^{\alpha}}{\Gamma(1-\gamma)} \int_{a}^{x}(x-t)^{-\gamma} F\left(-\alpha, 1+\beta-\gamma ; 1-\gamma ; \frac{x-t}{x-h}\right) g(t) d t
$$


Equation (4.4) shows that $f_{h}^{\gamma, \alpha, \beta}(x)$ and $g_{h}^{\gamma, \alpha, \beta}(x)$ differ by a constant $k$, that is $f_{h}^{\gamma, \alpha, \beta}(x)-g_{h}^{\gamma, \alpha, \beta}(x)=k$ for any $x \in[a, b]$. But $f_{h}^{\gamma, \alpha, \beta}(a)=0$ by $(4.2)$ and $g_{h}^{\gamma, \alpha, \beta}(a)=0$, because (4.3) is a solvable equation. Hence $k=0$ and

$$
\frac{(x-h)^{\alpha}}{\Gamma(1-\gamma)} \int_{a}^{x}(x-t)^{-\gamma} F\left(\alpha, 1+\beta-\gamma ; 1-\gamma ; \frac{x-t}{x-h}\right)[f(t)-g(t)] d t=0 .
$$

This is an equation of the form (1.1) and the uniqueness of its solution leads to the result $f(t)=g(t)$. The proof of Theorem 1 is complete.

\section{SUFFICIENT CONDITIONS FOR THE SOLVABILITY AND NEW FORM FOR THE SOLUTION}

The criterion of solvability of the Abel-type hypergeometric integral equation (1.1) was obtained in Theorem 1 in terms of the auxiliary function $f_{h}^{\gamma, \alpha, \beta}(x)$. The result below gives sufficient conditions in terms of the function $f(x)$ itself. To prove such a result, we need in the preliminary assertion contained in

Lemma 2. Let $f(x) \in A C([a, b]), h \leq a$ and let $\alpha, \beta$ and $\gamma$ be real numbers such that $0<\gamma<1$, when $h<a$, and

$$
0<\gamma<1, \gamma-\alpha-1<\beta<1+\alpha, \gamma<1+\alpha
$$

when $h=a$. Then $f_{h}^{\gamma, \alpha, \beta}(x) \in A C[a, b]$ and

$$
\begin{aligned}
& f_{h}^{\gamma, \alpha, \beta}(x)=\frac{(x-h)^{\alpha}(x-a)^{1-\gamma} f(a)}{\Gamma(2-\gamma)}{ }_{2} F_{1}\left(-\alpha, 1+\beta-\gamma ; 2-\gamma ; \frac{x-a}{x-h}\right) \\
& +\frac{(x-h)^{\alpha}}{\Gamma(2-\gamma)} \int_{a}^{x}(x-t)^{1-\gamma} F\left(-\alpha, 1+\beta-\gamma ; 2-\gamma ; \frac{x-t}{x-h}\right) f^{\prime}(t) d t .
\end{aligned}
$$

In particular, when $h=a$

$$
\begin{gathered}
f_{a}^{\gamma, \alpha, \beta}(x)=\frac{\Gamma(1+\alpha-\beta) f(a)}{\Gamma(2+\alpha-\gamma) \Gamma(1-\beta)}(x-a)^{\alpha-\gamma+1} \\
+\frac{(x-a)^{\alpha}}{\Gamma(2-\gamma)} \int_{a}^{x}(x-t)^{1-\gamma} F\left(-\alpha, 1+\beta-\gamma ; 2-\gamma ; \frac{x-t}{x-a}\right) f^{\prime}(t) d t .
\end{gathered}
$$

$\mathrm{P}$ r o o f. Since, by hypothesis, $f(t) \in A C[a, b]$, in view of $(4.1), f(t)$ is representable in the form:

$$
f(t)=f(a)+\int_{a}^{t} f^{\prime}(\tau) d \tau
$$


Substituting this relation into (3.1), we have

$$
\begin{gathered}
f_{h}^{\gamma, \alpha, \beta}(x)=\frac{(x-h)^{\alpha} f(a)}{\Gamma(1-\gamma)} \int_{a}^{x}(x-t)^{-\gamma} F\left(-\alpha, 1+\beta-\gamma ; 1-\gamma ; \frac{x-t}{x-h}\right) d t \\
+\frac{(x-h)^{\alpha}}{\Gamma(1-\gamma)} \int_{a}^{x}(x-t)^{-\gamma} F\left(-\alpha, 1+\beta-\gamma ; 1-\gamma ; \frac{x-t}{x-h}\right) d t \int_{a}^{t} f^{\prime}(\tau) d \tau \\
=I_{h}(x)+J_{h}(x) .
\end{gathered}
$$

According to (1.2), we evaluate $I_{h}(x)$ :

$$
\begin{gathered}
I_{h}(x)=\frac{(x-h)^{\alpha} f(a)}{\Gamma(1-\gamma)} \sum_{k=0}^{\infty} \frac{(-\alpha)_{k}(1+\beta-\gamma)_{k}}{(1-\gamma)_{k} k !}(x-h)^{-k} \int_{a}^{x}(x-t)^{k-\gamma} d t \\
=\frac{(x-h)^{\alpha}(x-a)^{1-\gamma} f(a)}{\Gamma(2-\gamma)} F\left(-\alpha, 1+\beta-\gamma ; 2-\gamma ; \frac{x-a}{x-h}\right)
\end{gathered}
$$

In particular, when $h=a$ and $1+\alpha-\beta>0$ we have

$$
I_{a}(x)=\frac{\Gamma(1+\alpha-\beta) f(a)}{\Gamma(2+\alpha-\gamma) \Gamma(1-\beta)}(x-a)^{\alpha-\gamma+1}
$$

in accordance with (3.7). As for $J_{h}(x)$, after interchanging the order of integration and evaluating the inner integral with using (2.2) we have

$$
\begin{gathered}
J_{h}(x)=\frac{(x-h)^{\alpha}}{\Gamma(1-\gamma)} \int_{a}^{x} f^{\prime}(\tau) d \tau \int_{\tau}^{x}(x-t)^{-\gamma} F\left(-\alpha, 1+\beta-\gamma ; 1-\gamma ; \frac{x-t}{x-h}\right) d t \\
=\frac{(x-h)^{\alpha}}{\Gamma(1-\gamma)} \int_{a}^{x}\left[\sum_{k=0}^{\infty} \frac{(-\alpha)_{k}(1+\beta-\gamma)_{k}}{(1-\gamma)_{k} k !}(x-h)^{-k} \int_{\tau}^{x}(x-t)^{-\gamma+k} d \tau\right] f^{\prime}(\tau) d \tau \\
=\frac{(x-h)^{\alpha}}{\Gamma(2-\gamma)} \int_{a}^{x}(x-t)^{1-\gamma} F\left(-\alpha, 1+\beta-\gamma ; 2-\gamma ; \frac{x-t}{x-h}\right) f^{\prime}(t) d t
\end{gathered}
$$

If $h<a$, it is clear, that $I_{h}(x), J_{h}(x) \in A C[a, b]$ and hence $f_{h}^{\gamma, \alpha, \beta}(x) \in$ $A C[a, b]$ in (5.2). When $h=a, I_{a}(x)$ in (5.7) is an absolutely continuous function, because

$$
(x-a)^{\alpha-\gamma-1}=(\alpha-\gamma+1) \int_{a}^{x}(t-a)^{\alpha-\gamma} d t
$$


and $(t-a)^{\alpha-\gamma} \in L_{1}(a, b)$ by the condition $\alpha-\gamma+1>0$ in (5.1). To prove that $J_{a}(x) \in A C([a, b])$, we first note that, in accordance with asymptotic behavior of $(1.2)$ at infinity $[3,2.3(9)]$,

$F\left(-\alpha, 1+\beta-\gamma ; 2-\gamma ; \frac{x-t}{x-a}\right)=O\left((x-a)^{-\alpha}\right)+O\left((x-a)^{1+\beta-\gamma}\right)(x \rightarrow a)$

for noninteger $\alpha+1+\beta-\gamma$, with addition of $\log (x-a)$ in the case of integer $\alpha+1+\beta-\gamma$. Therefore, $J_{a}(a)=0$, by the condition $\alpha+1+\beta-\gamma>0$ in (5.1). So we can represent $J_{a}(x)$ in the form

$$
J_{a}(x)=\int_{a}^{x} h(t) d t, h(x)=\frac{d}{d x} J_{a}(x)
$$

By using (1.2) and (1.3) and making term-by-term differentiation, being justified under he conditions in (5.1), it is easily seen that

$$
\begin{gathered}
h(x)=h_{1}(x)+h_{2}(x) \\
\equiv \frac{(x-a)^{\alpha}}{\Gamma(1-\gamma)} \int_{a}^{x}(x-t)^{-\gamma} F\left(-\alpha, 1+\beta-\gamma ; 1-\gamma ; \frac{x-t}{x-a}\right) f^{\prime}(t) d t \\
+\frac{\alpha(x-a)^{\alpha-1}}{\Gamma(2-\gamma)} \int_{a}^{x}(x-t)^{1-\gamma} F\left(1-\alpha, 1+\beta-\gamma ; 2-\gamma ; \frac{x-t}{x-a}\right) f^{\prime}(t) d t
\end{gathered}
$$

It is proved similarly to those in (3.8) and (3.9) that

$$
\int_{a}^{b} h_{1}(x) d x=\int_{a}^{b} g_{1}(t) d t, \int_{a}^{b} h_{2}(x) d x=\int_{a}^{b} g_{2}(t) d t
$$

where

$$
\begin{gathered}
g_{1}(t)=\frac{1}{\Gamma(2-\gamma)}(t-a)^{\alpha}(b-t)^{1-\gamma} F\left(-\alpha,-\beta ; 2-\gamma ;-\frac{b-t}{t-a}\right) f^{\prime}(t), \\
g_{2}(t)=\frac{1}{\Gamma(3-\gamma)}(t-a)^{\alpha-1}(b-t)^{2-\gamma} F\left(1-\alpha, 1-\beta ; 3-\gamma ;-\frac{b-t}{t-a}\right) f^{\prime}(t)
\end{gathered}
$$

and

$$
\int_{a}^{b}\left|g_{1}(t)\right| d t<\infty, \quad \int_{a}^{b}\left|g_{2}(t)\right| d t<\infty .
$$

Hence $h(x) \in L_{1}(a, b)$ and $J_{a}(x)$ is also an absolutely continuous function in accordance with (5.8) and (5.10) and $f_{a}^{\gamma, \alpha, \beta}(x) \in A C([a, b])$. 
The representation (5.2) follows from (5.4)-(5.6). This completes the proof of Lemma 2.

Corollary. Under the conditions of Lemma $2, f_{h}^{\gamma, \alpha, \beta}(a)=0$.

The following result gives a new form of the inversion formula for the equation (1.1) applicable to absolutely continuous functions.

THEOREM 2. Let $f(x) \in A C([a, b]), h \leq a$ and let $\alpha, \beta$ and $\gamma$ be real numbers such that the conditions in Lemma 2 are satisfied. Then the Abeltype hypergeometric equation (1.1) is solvable in $L_{1}(a, b)$ and its solution (2.3) can be expressed in the form:

$$
\begin{gathered}
\varphi(x)=\frac{(x-a)^{-\gamma}(x-h)^{\alpha}}{\Gamma(1-\gamma)} F\left(-\alpha, 1+\beta-\gamma ; 1-\gamma ; \frac{x-a}{x-h}\right) f(a) \\
+\alpha \frac{(x-a)^{1-\gamma}(x-h)^{\alpha-1}}{\Gamma(1-\gamma)} F\left(1-\alpha, 1+\beta-\gamma ; 2-\gamma ; \frac{x-a}{x-h}\right) f(a) \\
+\frac{(x-h)^{\alpha}}{\Gamma(1-\gamma)} \int_{a}^{x}(x-t)^{-\gamma} F\left(-\alpha, 1+\beta-\gamma ; 1-\gamma ; \frac{x-t}{x-h}\right) f^{\prime}(t) d t \\
+\frac{\alpha(x-h)^{\alpha-1}}{\Gamma(2-\gamma)} \int_{a}^{x}(x-t)^{1-\gamma} F\left(1-\alpha, 1+\beta-\gamma ; 2-\gamma ; \frac{x-t}{x-h}\right) f^{\prime}(t) d t .
\end{gathered}
$$

When $h=a$, this solution takes the form

$$
\begin{gathered}
\varphi(x)=\frac{\Gamma(1+\alpha-\beta) f(a)}{\Gamma(1+\alpha-\gamma) \Gamma(1-\beta)}(x-a)^{\alpha-\gamma} \\
+\frac{(x-a)^{\alpha}}{\Gamma(1-\gamma)} \int_{a}^{x}(x-t)^{-\gamma} F\left(-\alpha, 1+\beta-\gamma ; 1-\gamma ; \frac{x-t}{x-a}\right) f^{\prime}(t) d t \\
+\frac{\alpha(x-a)^{\alpha-1}}{\Gamma(2-\gamma)} \int_{a}^{x}(x-t)^{1-\gamma} F\left(1-\alpha, 1+\beta-\gamma ; 2-\gamma ; \frac{x-t}{x-a}\right) f^{\prime}(t) d t .
\end{gathered}
$$

$\mathrm{P}$ r o o f. By Lemma 2 and its corollary, $f_{h}^{\gamma, \alpha, \beta}(x) \in A C([a, b])$ and $f_{h}^{\gamma, \alpha, \beta}(a)=0$. So the conditions (4.2) of Theorem 1 are satisfied and the equation (1.1) is solvable in $L_{1}(a, b)$. Since $\varphi(x)=\left(f_{h}^{\gamma, \alpha, \beta}(x)\right)^{\prime},(5.12)$ is obtained by differentiating (5.2). The theorem is thus proved.

REMARK 1. The results in Lemmas 1 and 2 and Theorems 1 and 2 generalize the corresponding statements for the classical Abel integral equation studied in [22, Section 2.2].

REMARK 2. The results of Sections 4 and 5 given in Theorems 1 and 2, and in particular the new forms (5.12) and (5.13) for the solution of the equation (1.1), can be used to other similar types of integral equations involving modified and particular forms of Gauss hypergeometric function $F(a, b ; c ; z)$ (see 
[22] and [25]). For example, if we put $h=a$ in (1.1) and replace $\alpha$ by $\alpha+\beta$, $\gamma$ by $\alpha$ and $\beta$ by $-\eta$ in (3.1), we obtain the equation with the generalized fractional integral operator in the left-hand side introduced in [17]. Theorems 1 and 2 with the above spesializations yield new forms of results concerning the solvability of this equation and they can be applied to solve the boundary value problems where such equation arise (see [5], [13]-[16], [18]-[20], [21], [24], [26], [27]).

REMARK 3. The results in Theorem 1 leads to the definition of the generalized fractional integration and differentiation operators defined by the lefthand side of the equation (1.1) and the right-hand side of the equation (2.3), respectively. Theorem 2 gives sufficient conditions for the existence of such a generalized fractional derivative and its another forms (5.12) and (5.13).

\section{Acknowledgment}

The present investigation was supported, in part, by Belarusian Fundamental Research Fund and Department of Science and Technology (Government of India, Grant No. DST/MS/PM-001/93).

\section{REFERENCES}

[1] B.L.J. Braaksma and A. Schuitman. Some classes of Watson transforms and related integral equations for generalized functions, SIAM J. Math. Anal. 7(6), 1976, P. 771-796.

[2] Yu.A. Brychkov Yu., H.-J. Glaeske, A.P. Prudnikov, and Vu Kim Tuan. Multidimensional Integral Transformations, Gordon and Breach, New York, 1992.

[3] A. Erdelyi, W. Magnus, F. Oberhettinger and F.G. Tricomi. Higher Transcendental Functions, 1, McGraw-Hill, New York, 1953.

[4] T.P. Higgins. A hypergeometric integral transform, J. Soc. Industr. Appl. Math., 12, 1964, P. 601-612.

[5] A.A. Kilbas, M. Saigo, and O.A. Repin. Solution in closed form of boundary value problem for degenerate equation of hyperbolic type, Kyungpook Math. J., 36(2), 1996, P. 261-273.

[6] A.A. Kilbas, M. Saigo, and H. Takushima. On integrable solution of a multidimensional Abel-type integral equation, Fukuoka Univ. Sci. Rep., 25(1), 1995, P. $1-9$.

[7] E.R. Love. Some integral equations involving hypergeometric functions, Proc. Edinburgh Math. Soc. (2) 15(3), 1967, P. 169-198.

[8] E.R. Love. Two more hypergeometric integral equations, Proc. Cambridge Philos. Soc., 63(4), 1967, P. 1055-1076.

[9] E.R. Love. A hypergeometric integral equation, Lect. Notes. Math., 457, 1975, P. $272-288$.

[10] O.I. Marichev. Handbook of Integral Transforms and Higher Transcendental Functions. Theory and Algorithmic Tables, Ellis Horwood, Chichester, 1982.

[11] A.C. McBride. Solution of hypergeometric integral equation involving generalized functions, Proc. Edinburgh Math. Soc. (2) 19(3), 1975, P. 265-285.

[12] T.R. Prabhakar. A class of integral equations with Gauss functions in the kernels, Math. Nachr. 52(1-6), 1972, P. 71-83. 
[13] O.A. Repin. Boundary value problem for an equation of moisture transfer, Differtsial'nye Uravneniya 26(1), 1990, P. 169-171. (In Russian).

[14] O.A. Repin. Non-local boundary value problem for parabolic-hyperbolic equation with the charteristic line of change, Differentsial'nye Uravneniya, 28(2), 1992, P. 173-176. (In Russian).

[15] O.A. Repin. Boundary Value Problems with Shift for Equations of Hyperbolic and Mixed Type, Saratov Univ., Saratov, 1992. (In Russian)

[16] O.A. Repin. Non-local boundary value problem for one degenerate hyperbolic equation, Dokl. Akad. Nauk. Russ. Akad. Nauk, 335(3), 1994, P. 295-296. (In Russian)

[17] M. Saigo. A remark on integral operator involving the Gauss hypergeometric function, Math. Rep. Kyushu Univ., 11(2), 1978, P. 135-143.

[18] M. Saigo. A certain boundary value problem for the Euler-Darboux equation, Math. Japon., 24(4), 1979, P. 377-385.

[19] M. Saigo. A certain boundary value problem for the Euler-Darboux equation II, Math. Japon., 25(2), 1980, P. 211-220.

[20] M. Saigo. A certain boundary value problem for the Euler-Darboux equation III, Math. Japon., 26(1), 1981, P. 103-119.

[21] M. Saigo, O.A. Repin, and A.A. Kilbas. On a non-local boundary value problem for an equation of mixed parabolic-hyperbolic type, Internat. J. Math. Statist. Sci., 5(1), 1996, P. 1-16.

[22] S.G. Samko, A.A. Kilbas, and O.I. Marichev. Fractional Integrals and Derivatives. Theory and Applications, Gordon and Breach, New York, 1993.

[23] R.K. Saxena and R. Kumbhat. A generalization of Kober operators, Vijnana Parishad Anusandhan Patrica, 16(1), 1973, P. 31-36.

[24] M.M. Smirnov. A solution in closed form of the Volterra equation with a hypergeometric function in the kernel, Differentsial'nye Uravneniya, 18(1), 1982, P. 171-173. (In Russian)

[25] H.M. Srivastava and R.G. Buschman. Theory and Applications of Convolution Integral Equations, Kluwer, Boston, 1992.

[26] H.M. Srivastava and M. Saigo. Multiplication of fractional calculus operators and boundary value problems involving the Euler-Darboux equation, J. Math. Anal. Appl., 128(2), 1987, P. 325-369.

[27] V.F. Volkodavov and O.A. Repin. A boundary value problem for the Euler- Darboux equation with positive parameter, Differentsial'nye Uravneniya, 19(7), 1982, P. 1275-1277. (In Russian) 